16. J.F. Lafortune and M. Lccours, Measurement and modeling of propagation losses in a building at $900 \mathrm{MHz}$, IEEE Trans Veh Technol 39 (1990), 101-108.

17. S.W. Lee and G.A. Deschamps, A uniform asymptotic theory of EM diffraction by a curved wedge, IEEE Trans Antennas Propagat AP-24 (1976), 25-34.

18. R.J. Marhefka, NEC-Basic scattering code, user's manual (version 3.2), final rep 718422-4, The Ohio State University Electro Science Lab, Department of Electrical Engineering, Dec. 1990; prepared under Contract N60530-85-C-0249 for Naval Weapons Center.

19. R.L. LaFara, Computer method for science and engineering, Hayden, Rochelle Park, NJ, 1973, pp. 148-456.

(c) 2001 John Wiley \& Sons, Inc.

\section{E-POLARIZED BEAM SCATTERING BY AN OPEN CYLINDRICAL PEC STRIP HAVING AN ARBITRARY "CONICAL-SECTION" PROFILE}

\author{
Taner Oğuzer, ${ }^{1}$ Alexander I. Nosich, ${ }^{2}$ and Ayhan Altintaş ${ }^{3}$ \\ ${ }^{1}$ Department of Electrical and Electronics Engineering \\ Dokuz Eylul University \\ Buca 35160, Izmir, Turkey \\ ${ }^{2}$ Institute of Radio-Physics and Electronics \\ National Academy of Sciences \\ Kharkov 61085, Ukraine \\ and \\ LART \\ Universite de Rennes 1 \\ 35042 Rennes Cedex, France \\ ${ }^{3}$ Department of Electrical and Electronics Engineering \\ Bilkent University \\ 06533 Ankara, Turkey
}

\section{Received 26 June 2001}

\begin{abstract}
Two-dimensional (2-D) scattering of waves by a conducting strip with a canonical profile is simulated in the E-polarization case. This analysis is performed by reducing a singular integral equation (IE) to the dual-series equations, and making their analytical regularization. Furthermore, the incident field is taken as a complex source point (CSP) beam. This is an extension of our previous studies about circular and parabolic reflector antennas. The algorithm features are demonstrated. Far-field characteristics are presented for quite large-size curves strips of elliptic, parabolic, and hyperbolic profiles. (c) 2001 John Wiley \& Sons, Inc. Microwave Opt Technol Lett 31: 480-484, 2001.
\end{abstract}

Key words: wave scattering; conducting strip; electromagnetics; numerical methods

\section{INTRODUCTION}

The scattering of waves from a 2-D curves strip with a zero thickness is one of the traditional boundary-value problems in diffraction theory. The perfectly electrical conducting (PEC) case is the simplest type of this problem. An explicit solution to this problem is not possible, even for a PEC circular strip. However, analytical expressions can be obtained by using the asymptotic (high-frequency) approximations [1]. Another way is to obtain a numerical solution. For this purpose, one of the versions of the method of moments (MoM) can be used. In MoM, an electric-field integral equation (EFIE), which is obtained from the boundary condition, is discretized by approximating the unknown surface current density with a set of basis functions. Finally, the problem is converted to an algebraic matrix equation, and solved numerically. In this way, small and medium-size scatterers can be solved with a practical (a few digits) accuracy [2]. However, for larger geometries or better accuracy, the conventional MoM meets some intrinsic problems. This is because its convergence is not guaranteed in the mathematical sense, as an opportunity of minimization of computational errors [3].

Microwave reflectors are normally of several dozens of wavelengths in size. Therefore, an accurate and reliable numerical analysis of these scatterers should be done by using the method of analytical regularization (MAR) [4]. In the 2-D PEC strip analysis, this method is based on the inversion of the singular, namely, the static, part of the EFIE, with some special function-theoretic technique like the Riemann-Hilbert problem (RHP) [5, 6]. However the remaining part still exists, and cannot be analytically evaluated, which leads to an algebraic matrix equation. Therefore, this kind of technique is also called a semi-inversion method. As the resulting matrix equation has Fredholm second-kind properties, it can be solved numerically in an efficient manner, with a guarantee of the accuracy and convergence of the solution.

By using the strip scattering, 2-D models of reflector antennas can be analyzed, which has great practical importance, especially in modern communication systems. Reflector antennas are one of the best ways to communicate from one point to another with a minimum loss. Here, the most common method to feed a reflector is to illuminate it with a tapered beam radiated by a horn feed. We will simulate the feed by the CSP method [7]. This convenient tool was first applied in [8] to analyze a 2-D parabolic reflector antenna in combination with high-frequency techniques. Nevertheless, the latter are only valid for electrically very large structures, and furthermore, it is not possible to obtain a full pattern using a single method. Therefore, in [9], we have studied a 2-D circular PEC reflector antenna by combining CSP with MAR-RHP in one accurate technique.

However, when a reflector antenna is considered, the most realistic reflector profile is certainly a parabolic-shape surface. In [10], such a reflector was solved by following the idea first expressed in [11] - by using the MAR in the form of a modified RHP technique. Here, one of the basic steps is to introduce an auxiliary circle that is smoothly joined with a parabola, and exploit the semi-inversion of the wave scattering from a circularly curved strip, done by the RHP method. This approach has much in common with that of [12, 13], where semi-inversion was based on the extraction of the static part only, and on the usage of the Abel integral equation theory. Besides guaranteed convergence, electrically large scatterers are easily computed with the MAR due to the efficient computation of the Fourier series coefficients of nonsingular kernels by using the double fast Fourier transform (DFFT) algorithm. In this paper, the formulation of [10] is generalized for all so-called "conical-section" contours, which are the curves obtained as the sections of a cone by arbitrary plane: ellipse, parabola, and hyperbola. All of these curves can be represented by the same equation with different eccentricity factors $e$. We keep in mind that such a common formulation will be very useful in the solution of dual-reflector antenna systems.

\section{FORMULATION}

Cross sections of two problem geometries of infinitely thin PEC curved screens symmetrically illuminated by a directive feed are shown in Figures 1 and 2. The first one, as shown in 


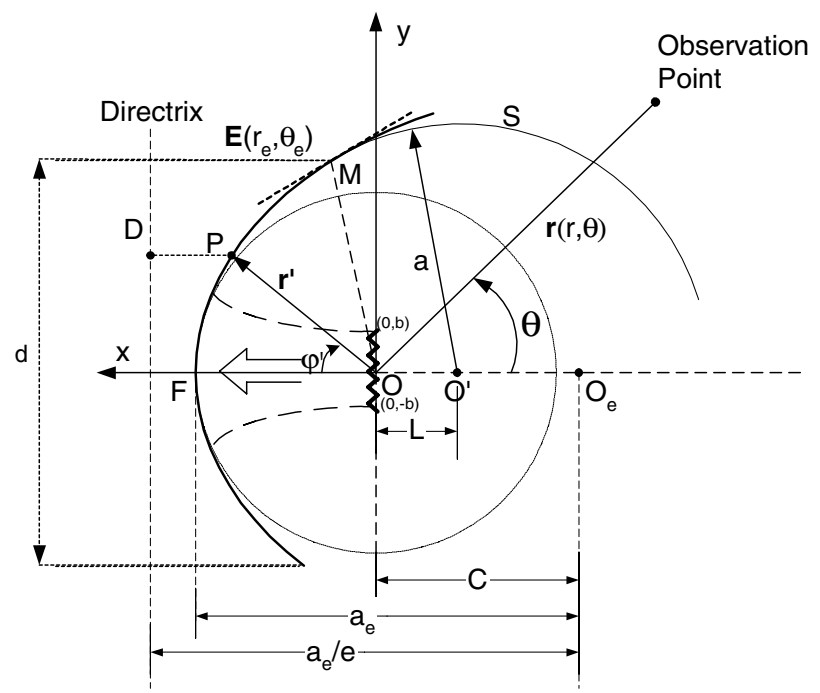

Figure 1 Geometry of the reflector antenna system with elliptic surface

Figure 1, is a 2-D contour with an elliptic-arc profile with the feed in a geometrical focus of the ellipse. The origin of the used coordinate system is taken just in this focus, and the point $O_{e}$ represents the symmetry center of the ellipse. Further, the focal distance $f$ is taken as a fixed value, i.e., $a_{e}-c=f$, while the curvature is assumed to increase from that of a circle to infinity. The second geometry, shown in Figure 2, is similar to the first one, but has a hyperbolic-arc strip profile, and the feed is in a geometrical focus of hyperbola. In this case, the symmetry point $O_{h}$ appears to the left of the strip. The focus $f$ of the hyperbola is assumed to be a fixed value $f$ as given by $c-a_{h}=f$. Both of these curves can be represented by the same equation, namely,

$$
y^{2}+\left(1-e^{2}\right) x^{2}+2 f e(1+e) x=f^{2}\left(1+e^{2}\right)
$$

where $e=c / a_{e, h}$ is the eccentricity factor of the curve, and defines a circle $(e=0)$, ellipse $(0<e<1)$, parabola $(e=1)$, or hyperbola $(1<e<\infty)$. For the ellipse, the semiaxes in the $x$ - and $y$-directions can be expressed as $a=f /(1-e)$ and $b=f \sqrt{(1+e) /(1-e)}$, respectively. When building the so-

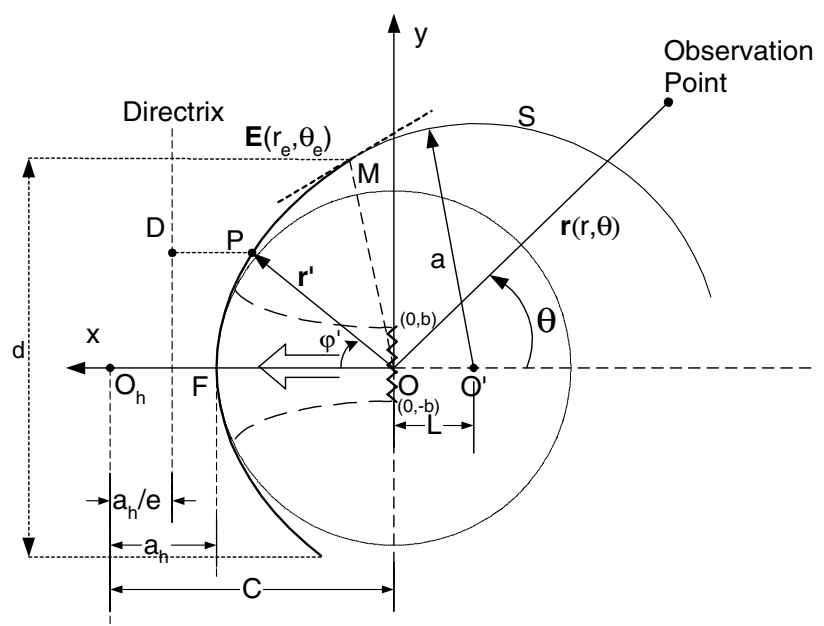

Figure 2 Geometry of the reflector antenna system with hyperbolic surface lution, an open arc of this generalized curve (representing the scatterer cross section) is completed to the closed contour $C$ by a circle having its origin on the $x$-axis. Its radius is chosen in such a way that, at the connection points (i.e., the arc's endpoints) $E\left(r_{e}, \theta_{e}\right)$, the curvatures of the arc and the circle are matched (taken the same). As a result, the contour first derivatives are continuous, and discontinuities in the second derivatives are finite. As we will see, this twice-continuous closed contour $C(M U S)$ is smooth enough to develop a regularized solution to the formulated problem. Another condition for the solvability of the problem is that the branch cut associated with the CSP in the real space must not cross the strip contour $M$ (see [9]).

The requirements for the rigorous formulation of the considered boundary-value problem can be stated as the satisfaction of the Helmholtz equation, Sommerfeld radiation condition in the far zone, the PEC boundary condition on the reflector cross-section contour $M$, and the edge condition at its endpoints. As known, the free-space Green's function in 2-D, i.e., $G_{0}^{E}=i / 4 H_{0}^{(1)}\left(k_{0}\left|\vec{r}(\phi)-\vec{r}^{\prime}\left(\phi^{\prime}\right)\right|\right)$, satisfies both the Helmholtz equation and the radiation condition. Then the basic EFIE can be obtained by applying the Dirichlet-type boundary condition, valid in the $E$-polarization case, to a single-layer potential representation of the scattered-field function, with the Green's function as a kernel:

$$
\int_{M} J_{z}\left(\vec{r}^{\prime}\right) G_{0}^{E}\left(\vec{r}, \vec{r}^{\prime}\right) d l^{\prime}=-E_{z}^{\mathrm{inc}}(\vec{r}), \quad \vec{r} \in M
$$

Here, $E_{z}^{\text {inc }}(\vec{r})$ is the known incident-field function, and $J_{z}(\vec{r})$ is an unknown surface-current density function. Assume that the closed smooth surface $C$ can be characterized by parametric equations $x=x(\varphi), y=y(\varphi), 0 \leq \varphi \leq 2 \pi$. Define the current density function to be 0 on the part of $C$ complementary to $M$. Then (2) becomes

$\int_{0}^{2 \pi} J_{z}\left(\varphi^{\prime}\right) G_{0}^{E}\left(\varphi, \varphi^{\prime}\right) \rho\left(\varphi^{\prime}\right) d \varphi^{\prime}=-E_{z}^{\mathrm{inc}}(\varphi), \quad s \in M$

where $\rho\left(\varphi^{\prime}\right)$ stands for Jacobian. A further idea is to use the entire-period (in $\varphi$ ) radial exponents as a set of global basis functions. Then, the Green's function $G_{0}^{E}\left(\varphi, \varphi^{\prime}\right)$ is to be expanded in terms of the double Fourier series. However, this is not enough to regularize our problem, so a new function is introduced as follows:

$$
\begin{aligned}
H\left(\varphi, \varphi^{\prime}\right)= & H_{0}^{(1)}\left(k_{0}\left|\vec{r}(\varphi)-\vec{r}^{\prime}\left(\varphi^{\prime}\right)\right|\right) \\
& -H_{0}^{(1)}\left(2 k_{0} a_{s} \sin \frac{\left|\varphi-\varphi^{\prime}\right|}{2}\right) \\
= & \sum_{m, n=-\infty}^{\infty} h_{n m} e^{i n \varphi} e^{i m \varphi^{\prime}}
\end{aligned}
$$

where $a_{s}$ represents the auxiliary circle radius.

In this way, the singularity of $H\left(\varphi, \varphi^{\prime}\right)$ is extracted from the kernel; however, the central point of an efficient semi-inversion technique is that, to ensure the smooth junction of the open $\operatorname{arc} M$ with the auxiliary circle, the latter radius is taken equal to $f$. Then the function $H\left(\varphi, \varphi^{\prime}\right)$ and its first derivatives with respect to $\varphi$ and $\varphi^{\prime}$ are continuous functions on $C$. Furthermore, the second derivative of $H\left(\varphi, \varphi^{\prime}\right)$, i.e., $\partial^{2} H\left(\varphi, \varphi^{\prime}\right) / \partial \varphi \partial \varphi^{\prime}$, has only a logarithmic singularity as $\varphi$ $\rightarrow \varphi^{\prime}=\varphi_{e}$. Hence, it is not continuous, but belongs to $L_{2}(C)$, and therefore the Fourier series coefficients $h_{n m}$ satisfy the 


$$
\sum_{m, n=-\infty}^{\infty}\left(|n|^{2}+1\right)\left(|m|^{2}+1\right)\left|h_{n m}\right|^{2}<\infty
$$

To discretize IE (3), all of the other functions are also expressed in terms of their Fourier series, assuming that this is justified:

$$
J_{z}\left(\varphi^{\prime}\right) \rho\left(\varphi^{\prime}\right)=\frac{2}{i \pi} \sum_{n=-\infty}^{\infty} x_{n}^{E} e^{i n \varphi^{\prime}}
$$

and

$$
E_{z}^{\mathrm{inc}}(\varphi)=\sum_{n=-\infty}^{\infty} b_{n}^{E} e^{i n \varphi}
$$

In the case of the CSR illumination, the right-hand-part coefficients are

$$
b_{n}^{E}=\frac{i}{8 \pi} \int_{0}^{2 \pi} H_{0}^{(1)}\left(k\left|\vec{r}(\varphi)-\vec{r}_{s}\right|\right) e^{-i n \varphi} d \theta
$$

and the complex source position is given as

$$
\vec{r}_{s}=i\left(b \cos \beta \hat{a}_{x}+b \sin \beta \hat{a}_{y}\right) .
$$

Here, parameters $b$ and $\beta$ are the beam width and beam-aiming angle of the directive horn source simulated with the aid of CSP [7-9]. Then, all of the Fourier expansions are substituted into (3) and, with the absence of the current on the aperture part of $C$, together constitute the following dual-series equations:

$$
\begin{gathered}
\sum_{n=-\infty}^{\infty}\left(x_{n}^{E} J_{n}\left(k_{0} a_{s}\right) H_{n}^{(1)}\left(k_{0} a_{s}\right)+\sum_{l=-\infty}^{\infty} h_{-l n} x_{l}^{E}\right) e^{i n \varphi} \\
=-\sum_{n=-\infty}^{\infty} b_{n}^{E} e^{i n \varphi}, \quad \varphi \in M \\
\sum_{n=-\infty}^{\infty} x_{n}^{E} e^{i n \varphi}=0, \quad \varphi \in S .
\end{gathered}
$$

To convert (10) and (11) into a certain convenient form, we denote a new set of unknown coefficients:

$$
y_{n}=x_{n}^{E} J_{n}\left(k_{0} a_{s}\right) H_{n}^{(1)}\left(k_{0} a_{s}\right)+\sum_{l=-\infty}^{\infty} h_{-l n} x_{l}^{E}+b_{n}^{E}
$$

and then obtain

$$
\begin{gathered}
\sum_{n=-\infty}^{\infty} y_{n} e^{i n \varphi}=0, \quad \varphi \in M \\
\sum_{n=-\infty}^{\infty} y_{n}|n| e^{i n \varphi}=\sum_{n=-\infty}^{\infty} f_{n} e^{i n \varphi}, \quad \varphi \in S
\end{gathered}
$$

where

$$
f_{n}=y_{n} \Delta_{n}-\frac{i}{\pi} \frac{b_{n}^{E}+\sum_{l=-\infty}^{\infty} x_{l}^{E} h_{-l n}}{J_{n}\left(k_{0} a_{s}\right) H_{n}^{(1)}\left(k_{0} a_{s}\right)}
$$

$$
\Delta_{n}=\frac{i}{\pi} \frac{1}{J_{n}\left(k_{0} a\right) H_{n}^{(1)}\left(k_{0} a\right)}+|n| .
$$

The given canonical-form dual-series equations can be converted to an infinite algebraic matrix equation of the Fredholm second kind by the application of the RHP technique in the form given in $[5,6]$. That is due to the following analytical solution to (13), (14), provided that $f_{n}, n=0$, $\pm 1, \ldots$ are known

$$
y_{m}=\sum_{n=-\infty}^{\infty} f_{n} \tilde{T}_{m n}
$$

where $\tilde{T}_{m n}=(-1)^{m+n} T_{m n}\left(-\cos \theta_{e}\right)$, and $T_{m n}$ functions can be found in $[5,6,9]$ as combinations of the Legendre polynomials. Finally, after substitution of $y_{n}$ in terms of $\hat{x}_{n}$ where $\hat{x}_{n}=x_{n} /(|n|+1)$, the resultant matrix equation becomes

$$
\left[\bar{I}+\bar{A}_{m l}^{1}-\bar{A}_{m l}^{2}-\bar{A}_{m l}^{3}\right] \hat{x}_{l}=\bar{B}_{m}
$$

where

$$
\begin{gathered}
A_{m l}^{1}=h_{-l m}(|l|+1) / Q_{m} \\
A_{m l}^{2}=J_{l}\left(k_{o} a_{s}\right) H_{l}^{(1)}\left(k_{o} a_{s}\right)(|l|+1) \Delta_{l} \tilde{T}_{m l} / Q_{m} \\
A_{m l}^{3}=(|l|+1) G_{l m} / Q_{m} \\
B_{m}=\sum_{n=-\infty}^{\infty}\left(|n| b_{n}^{E} \tilde{T}_{m n}-b_{m}^{E}\right) / Q_{m} \\
Q_{m}=(|m|+1) J_{m}\left(k_{0} a_{s}\right) H_{m}^{(1)}\left(k_{0} a_{s}\right), G_{l m}=\sum_{n=\infty}^{\infty} h_{-l n} \tilde{T}_{m n} .
\end{gathered}
$$

\section{NUMERICAL RESULTS}

The performed formulation is examined by the various numerical results related to the beam-forming characteristics of several reflectors. To do this, the "conical-section" reflector surface is modeled by the eccentricity factor $e$. The expansion coefficients for the right-hand-part (7) and the smooth kernel (4) were computed by using the FFT and DFFT algorithms, respectively. Figure 3 presents the total tangential electric field and induced surface current density, both on the metal and aperture part of the closed contour $C$, as a function of the arc length. It is understood from these plots that the required boundary conditions are satisfied. Figure 4 gives the truncation error (see [9] for the definition of this quantity) versus truncation number $N_{t r}$ in logarithmic scale for different eccentricity values $e$ and a fixed aperture dimension $(d)$. It is seen that the $N_{t r}$ required for reasonable accuracy increases with the $e$-factor. This is because the radius of the auxiliary circle smoothly completing arc $M$ to the closed contour $C$ increases by increasing the $e$-factor, although the aperture dimension of $M$ remains the same. Besides, some radiation pattern samples for the different $e$-values are presented in Figure 5 for fixed values of $d$ and the source directivity parameter $k b$. Naturally, the narrowest beamwidth is obtained in the parabolic case, i.e., $e=1$, and the lowest backlobe levels occurs for $e=0.5$. This is due to the fact that the edge illumination is reduced by decreasing the $e$-factor under the same $d$-value. Figure 6 shows the directivity versus 


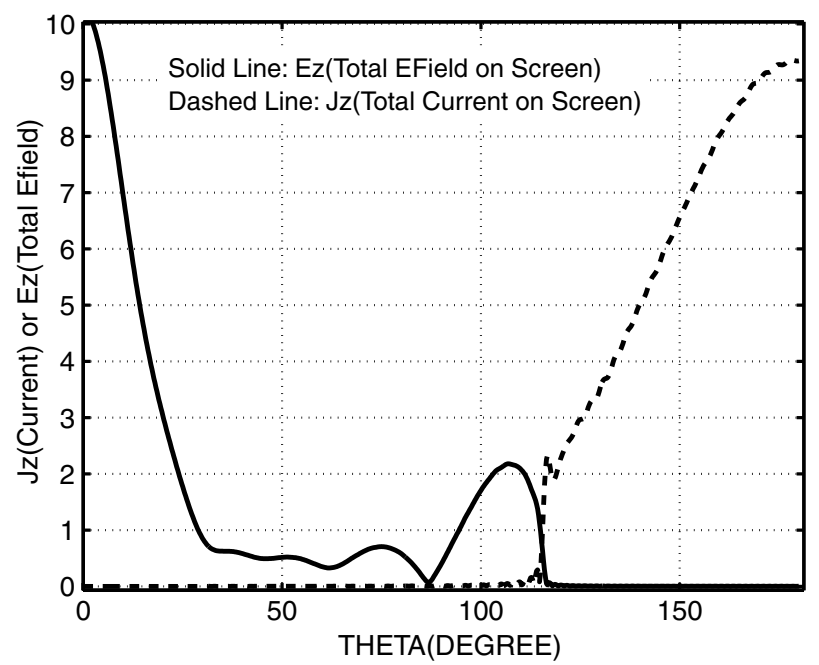

Figure 3 Current density and total tangential electric field distribution on the whole closed smooth surface $(f=2 \lambda, D=5 \lambda, k b=2.6$, and $e=1$ ). The corresponding edge illumination is $-13.92 \mathrm{~dB}$

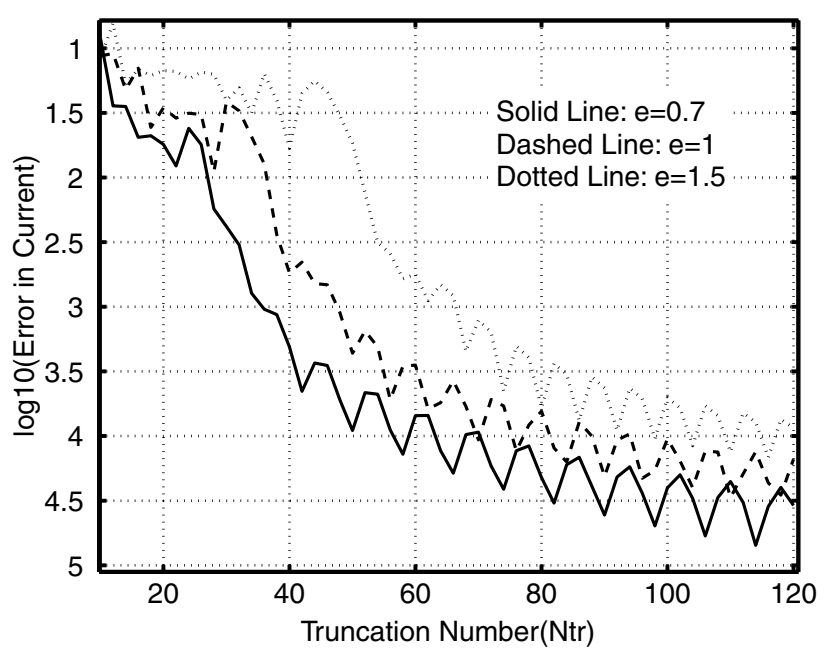

Figure 4 Relative accuracy of the truncated Fourier series coefficients of the current density $(f=2 \lambda, D=5 \lambda, k b=2.6$, and $e=0.7$, 1 , and 1.5)

the $e$-factor variation for the considered generalized reflector antenna. It is again seen that the maximum directivity is obtained in the parabolic case, i.e., at $e=1$. Finally, Figure 7 is a test of the physical-optics (PO) solution confronted with our accurate results performed for quite a large-size geometry. From the figure, it is observed that the presented and PO solutions coincide in the main beam, first sidelobes, and penumbra regions.

\section{CONCLUSION}

In the 2-D curved strip scattering simulations, arbitrary profile cylindrical PEC surfaces have been solved by the RHPbased regularization technique in the $E$-polarization case. The DFFT algorithm has been used in the computation of the resulting matrix elements that enabled us to solve, with a controlled accuracy, larger geometries than those presented in the literature. Efficient numerical solutions for the generalized "conical-section" profiles have been obtained in the case of a directive incident field, to study the effect of the

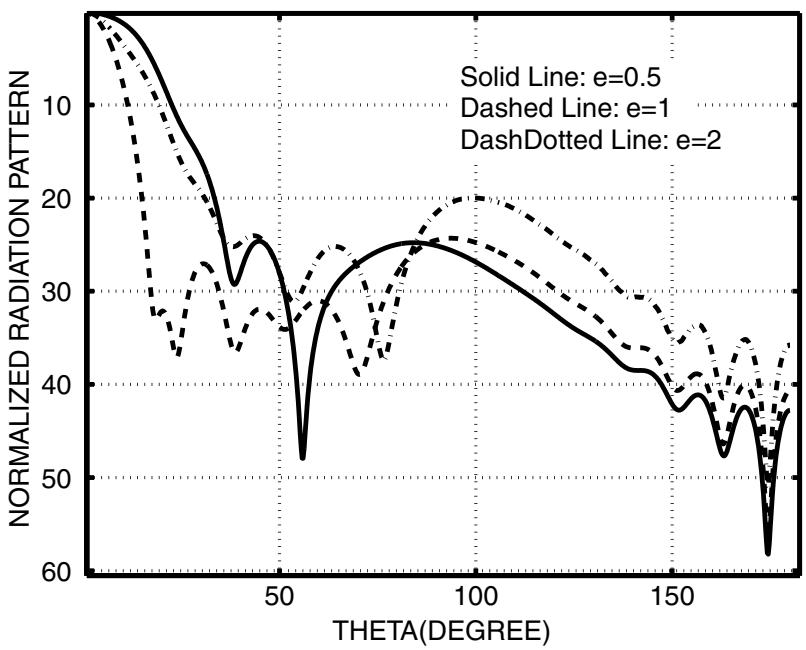

Figure 5 Radiation patterns for different eccentricity values $(e=$ $0.5,1$, and 2) under the same $f$ - and $D$-values, i.e., $f=2 \lambda, D=5 \lambda$, and $k b=2.6$

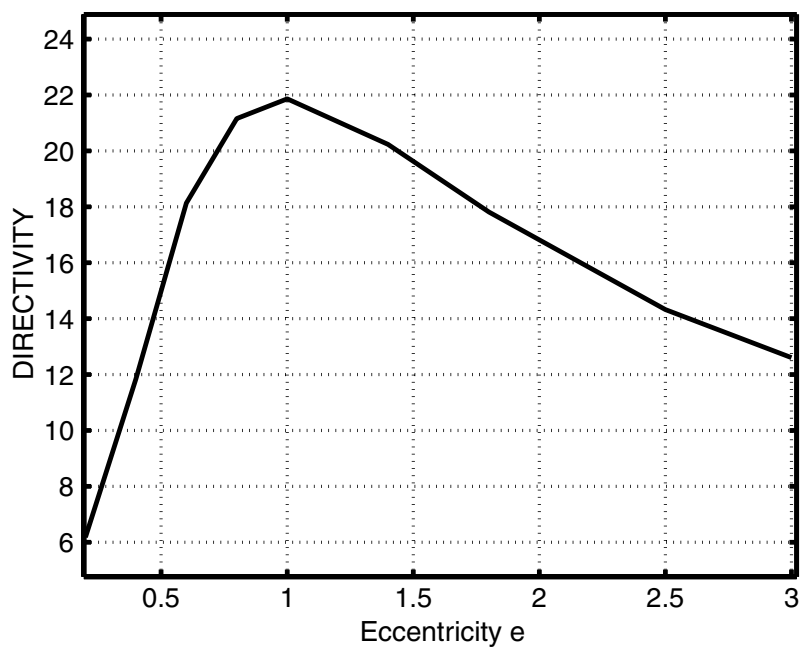

Figure 6 Directivity variation of the reflector antenna system with the eccentricity $(e)$-value and $f=2 \lambda, D=4 \lambda$, and $k b=2.6$

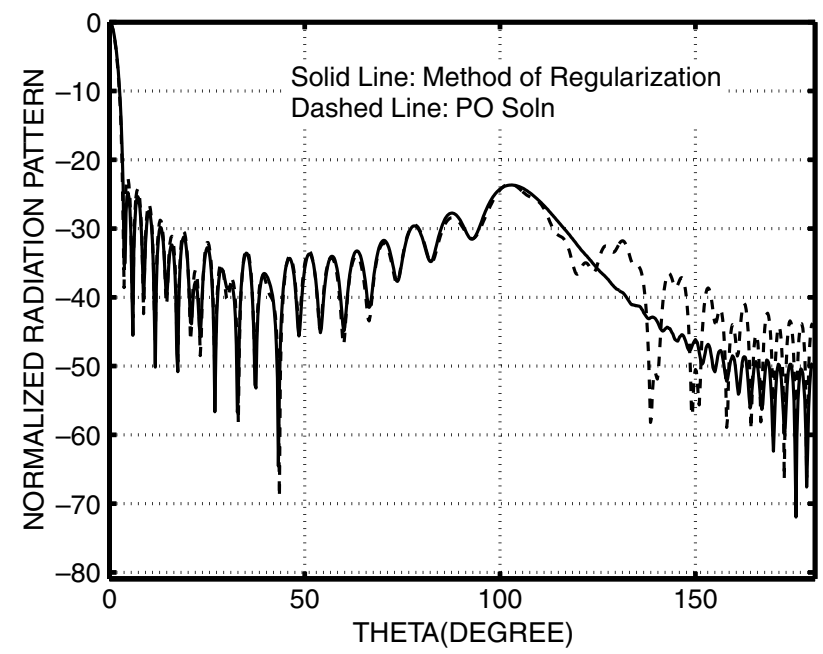

Figure 7 Comparison of the two radiation patterns obtained by the present method and physical optics and $f=8 \lambda, D=20 \lambda, k b=1.8$, and eccentricity $e=1$ (parabola). The corresponding edge illumination is $-10.21 \mathrm{~dB}$ 
strip shape on the beamforming. This will be used as a basis in follow-on dual-reflector antenna simulations.

\section{REFERENCES}

1. M. Idemen and A. Büyükaksoy, High frequency surface currents induced on a perfectly conducting cylindrical reflector, IEEE Trans Antennas Propagat AP-32 (1984), 501-507.

2. J.R. Mautz and R.F. Harrington, Electromagnetic penetration into a conducting circular cylinder through a narrow slot, TM case, J Electromag Waves Appl 2 (1988), 269-293.

3. D.G. Dudley, Error minimization and convergence in numerical methods, Electromag 5 (1985), 89-97.

4. A.I. Nosich, MAR in the wave-scattering and eigenvalue problems: Foundations and review of solutions, IEEE Antennas Propagat Mag 42 (1999), 34-49.

5. A.I. Nosich, "Green's function-Dual series approach in wave scattering from combined resonant scatterers," Analytical and numerical methods in electromagnetic wave theory, $\mathrm{M}$. Hashimoto et al. (Editors), Science House, Tokyo, Japan, 1993, pp. 419-469.

6. A. Altintaş and A.I. Nosich, "The method of regularization and its application to some electromagnetic problems," NATO-ASI: Computational electromagnetics, N. Uzunoglu (Editor), Springer-Verlag, Berlin, Germany, 1999.

7. L.B. Felsen, Complex source point solutions of the field equations and their relation to the propagating and scattering of Gaussian beams, Symp Math 18 (1976), 39-56.

8. G.A. Suedan and E.V. Jull, Beam diffraction by planar and parabolic reflectors, IEEE Trans Antennas Propagat 39 (1991).

9. T. Oğuzer, A. Altintaş, and A.I. Nosich, Accurate simulation of reflector antennas by complex source-Dual series approach, IEEE Trans Antennas Propagat 43 (1995), 793-802.

10. T. Oğuzer, A.I. Nosich, and A. Altintaş, Radiation characteristics of 2D parabolic microwave reflector antenna systems analyzed by complex source-dual series approach, Proc Int Symp Phys and Eng of MM and Sub-MM Waves (MSMW-01), Kharkov, Ukraine, 2001.

11. A.I. Nosich, Reflector antenna simulations by complex sourcedual series approach, Proc Int Conf MM Wave and Far-Infrared Sci and Technol (ICMWFST-94), Guangzhou, P.R. China, 1994, pp. 15-17.

12. Y.A. Tuchkin, Wave scattering by an open cylindrical screen of arbitrary profile with Dirichlet boundary value condition, Sov Phys Dok 30 (1985), 1027-1030.

13. Y.A. Tuchkin, Analytical regularization method for E-polarized electromagnetic wave diffraction by arbitrary shaped cylindrical obstacles, Proc Int Conf Math Methods in EM Theory (MMET*98), Kharkov, Ukraine, 1998, pp. 733-735. absorbing substrate coated with a single thin-film layer. The PPRBS operates in the UV region. The power reflection coefficient ranges from 69 to $90 \%$ for angles of incidence ranging from 85 to $89^{\circ}$, respectively. (C) 2001 John Wiley \& Sons, Inc. Microwave Opt Technol Lett 31: 484-487, 2001.

Key words: polarization preserving; reflected beam splitter; single-layer coating; absorbing substrate; $U V$ range

\section{INTRODUCTION}

Polarization-preserving devices are often needed in optical systems to preserve the polarization state of the incoming light after it goes through reflection or transmission. A number of polarization-preserving devices are reported in the literature. These devices consist of two parallel or orthogonal reflected substrates coated with appropriate thin-film layers [1-3]. In these devices, the incoming beam has to reflect off two substrates before it can return back to its original polarization. In this paper, we introduce the polarization-preserving reflected beam splitter (PPRBS). The PPRBS splits the incoming beam into two beams with two different directions. The state of polarization of the two beams is the same as the incoming beam. The advantage that the PPRBS has over the previously reported devices is that the PPRBS preserves the polarization of the incoming beam without the need to go through a second reflection.

The PPRBS consists of a V-shaped absorbing substrate coated with a single thin-film layer. The designed PPRBS operates in the ultraviolat (UV) region. Two different materials are considered as a substrate for the PPRBS. The first material is germanium (Ge), with a complex reflection index $N=2.516-j 4.669$ at a wavelength $\lambda=4.4 \mathrm{eV}$ or $281.93 \mathrm{~nm}$. The second material is gallium antimonide (GaSb), with a complex reflection index $N=2.522-j 4.13$ at a wavelength $\lambda=4.2 \mathrm{eV}$ or $295.35 \mathrm{~nm}$ [4]. The power reflection coefficient of the PPRBS ranges from 69 to $90 \%$ for an angle of incidence ranging from 85 to $89^{\circ}$.

\section{PROBLEM STATEMENT}

Consider the structure shown in Figure 1. The structure consists of a V-shaped absorbing substrate of a complex index of refraction $N_{2}=n_{2}-j k_{2}$. The substrate is placed in a medium of refractive index $N_{0}$. In this paper, we use $N_{0}=1$ for air. Both surfaces of the substrate are coated with a single

\section{POLARIZATION-PRESERVING REFLECTED BEAM SPLITTER}

\section{Mohamad A. Habli ${ }^{1}$}

${ }^{1}$ Information Engineering Department

Sultan Qaboos University

Al-Khod, Muscat 123, Sultanate of Oman

Received 6 July 2001

ABSTRACT: The design of a polarization preserving reflected beam splitter (PPRBS) is presented. The PPRBS consists of a V-shaped

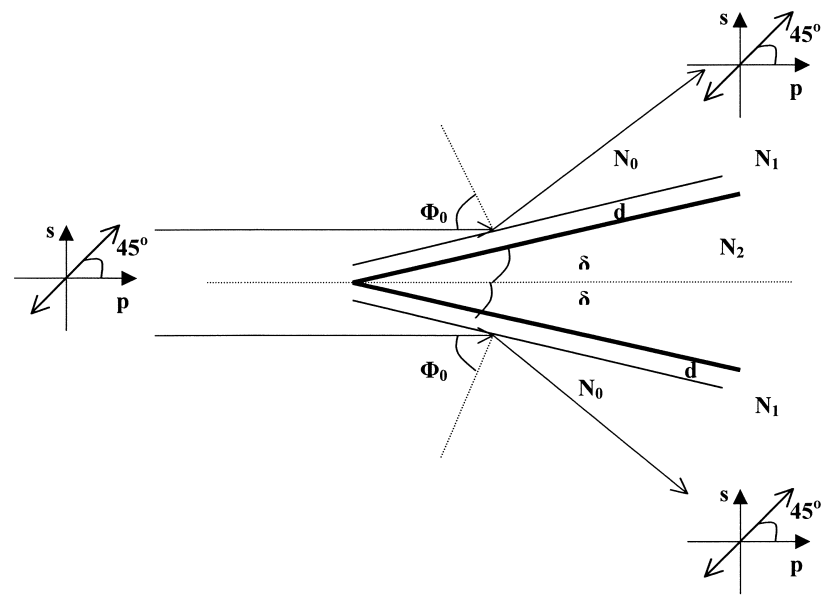

Figure 1 PPRBS layout 